\title{
Association between body mass index and newly diagnosed drug-resistant pulmonary tuberculosis in Shandong, China from 2004 to 2019
}

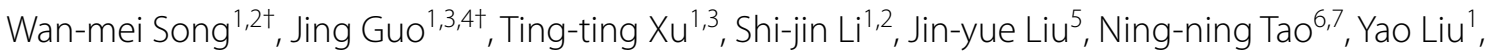
Qian-yun Zhang ${ }^{1,2}$, Si-qi Liu' ${ }^{1,2}$, Qi-qi An ${ }^{1,2}$, Yi-fan Li ${ }^{1}$, Chun-bao Yu ${ }^{8}$, Ji-hua Dong ${ }^{*^{*}}$ and Huai-Chen $\mathrm{Li}^{1,10^{*}}$

\begin{abstract}
Background: Drug-resistant tuberculosis (DR-TB), obesity, and malnutrition are growing public health problems in the world. However, little has discussed the impact of different BMI status on the emergence of TB drug resistance. We aimed to explore the drug-resistant profiles of DR-TB and its clinical predictors among underweight, overweight or obesity population.

Methods: 8957 newly diagnosed TB cases with drug susceptibility results and BMI data in Shandong China, from 2004 to 2019 were enrolled. Multivariable and univariable logistic regression models were applied to investigate the impact of BMI on different drug-resistance. Clinical predicators and drug-resistant profiles of DR-TB among obesity, underweight, normal TB group were also described.

Results: Among 8957 TB cases, 6417 (71.64\%) were normal weight, 2121 (23.68\%) were underweight, 373 (4.16\%) were overweight, and $46(0.51 \%)$ were obese. The proportion of drug resistance and co-morbidity among normal weight, underweight, overweight, obese TB groups were 18.86\%/18.25\%/20.38\%/23.91\% (DR-TB), 11.19\%/11.74\%/9.65\%/17.39\% (mono-resistant tuberculosis, MR-TB), 3.41\%/3.06\%/5.36\%/0.00\% (multidrug resistant tuberculosis, MDR-TB), 4.21\%/3.39\%/5.36\%/6.52\% (polydrug resistant tuberculosis, PDR-TB), 10.57\%/8.44\%/19.57\%/23.91\% (co-morbidity), respectively. Compared with normal weight group, underweight were associated with lower risk of streptomycin-related resistance (OR $0.844,95 \% \mathrm{Cl} 0.726-0.982)$, but contributed to a higher risk of MR-TB (isoniazid) (odds ratio (OR) 1.347, 95\% Cl 1.049-1.730; adjusted OR (aOR) 1.31, 95\% Cl 1.0171.686), $\mathrm{P}<0.05$. In addition, overweight were positively associated with MDR-TB (OR 1.603, 95\% Cl 1.002-2.566; aOR $1.639,95 \% \mathrm{Cl} 1.02-2.633)$, isoniazid + rifampicin + streptomycin resistance (OR 1.948, 95\% confidence interval (Cl):
\end{abstract}

\footnotetext{
*Correspondence: dongjihua321@sohu.com; lihuaichen@163.com

tWan-mei Song and Jing Guo contributed equally to this work

${ }^{1}$ Department of Respiratory and Critical Care Medicine, Shandong Provincial Hospital Affiliated to Shandong University, Shandong Provincial

Hospital Affiliated to Shandong First Medical University, Jinan 250021,

Shandong, People's Republic of China

${ }^{9}$ Department of Respiratory Medicine, Heze Mudan People's Hospital,

Heze 274000, Shandong, People's Republic of China

Full list of author information is available at the end of the article
}

(C) The Author(s) 2021. Open Access This article is licensed under a Creative Commons Attribution 4.0 International License, which permits use, sharing, adaptation, distribution and reproduction in any medium or format, as long as you give appropriate credit to the original author(s) and the source, provide a link to the Creative Commons licence, and indicate if changes were made. The images or other third party material in this article are included in the article's Creative Commons licence, unless indicated otherwise in a credit line to the material. If material is not included in the article's Creative Commons licence and your intended use is not permitted by statutory regulation or exceeds the permitted use, you will need to obtain permission directly from the copyright holder. To view a copy of this licence, visit http://creativecommons.org/licenses/by/4.0/. The Creative Commons Public Domain Dedication waiver (http://creativeco mmons.org/publicdomain/zero/1.0/) applies to the data made available in this article, unless otherwise stated in a credit line to the data. 
1.061-3.577; aOR 2.113,95\% Cl 1.141-3.912), Any isoniazid + streptomycin resistance (OR 1.472, 95\% Cl 1.013-2.14; aOR 1.483, 95\% Cl 1.017-2.164), $P<0.05$.

Conclusions: The higher risk of MDR-TB, isoniazid + rifampicin + streptomycin resistance, Any isoniazid + streptomycin resistance, and co-morbidity among overweight population implies that routine screening for drug sensitivity and more attention on co-morbidity among overweight TB cases may be necessary. In addition, underweight TB cases have a higher risk of isoniazid resistance. Our study suggests that an in-depth study of the interaction between host metabolic activity and infection of DR-TB may contribute more to novel treatment options or preventive measures, and accelerate the implementation of the STOP TB strategy.

Keywords: Tuberculosis, Drug resistance, Body mass index, Overweight, Underweight

\section{Background}

Tuberculosis (TB), is caused by Mycobacterium tuberculosis (MTB), contributes to a largest number of deaths from infectious diseases (more than HIV/AIDS) [1, 2]. According to WHO Tuberculosis Report, approximately 10.0 million population suffered from TB globally in 2018 , and the prevalence was 132 cases per 100,000 people [1]. Additionally, nearly 1.2 million HIV-negative and 251, 000 HIV-positive patients died from TB in 2018 [1]. Nine percent of the overall TB burden worldwide were from China, only second to India (27\%) [1].

Multidrug-resistant tuberculosis (MDR-TB) is defined as TB with resistance to at least isoniazid (INH) and rifampin (RFP), which is usually associated with longer hospitalization, more expensive treatment, and higher mortality [3]. The bacteriological cause is the transmission of MDR-TB strains in new cases or the acquired resistance through de novo mutation during TB treatment $[2,3]$. The emergence and spread of rifampin-resistant (RR-TB) or MDR-TB has brought grim challenge to TB control, and threatens the health of human being. The estimated proportions of RR-TB/MDR-TB among previously treated and newly diagnosed cases were $18 \%$ (95\% confidence interval [CI] 7.6-31\%) and 3.4\% (95\% CI 2.54.4\%) respectively [1]. Despite recent advances in early diagnosis of MDR-TB such as GeneXpert MTB/RIF, laboratories in many low-income countries are still unable to carry out routine bacterial culture and drug susceptibility testing (DST), thus clinical predictors of DR-TB especially MDR-TB may contribute to the early discovery and timely treatment of suspected cases $[4,5]$. The identification of clinical risk factors for the development of drug-resistant tuberculosis (DR-TB) will also benefit the management of TB patients and facilitate tuberculosis control programs $[1,5]$.

The critical influences of diverse nutritional status on TB infection have been recognized for decades [6]. Recent years, there were more and more researches focused on body mass index (BMI, usually used as one of the indicators to judge nutritional status) and $\mathrm{TB}$, for example, the impact of BMI on TB incidence, TB treatment outcomes, TB-related mortality, or negative conversion rate of new sputum among MDR-TB patients [7-9]. Lower BMI is a recognized risk factor for active tuberculosis infection [10]. The main causes of malnutrition were poverty and food shortages $[7,8]$. Malnutrition could damage the immunity of human body by decreasing the concentration of T-cell subset (including T-cytotoxic-suppressor cell, $\mathrm{T}$ helper cells, and natural killer cells), immunoglobulins, and interleukin-2 receptor, making them more vulnerable to infections such as TB and HIV [11]. Although, former researches revealed that the increased incidence of different infections such as hospital-acquired or postoperative infections might be attributable to obesity [12], available evidence on obesity and risk of active tuberculosis infection indicated an inverse relationship [6]. Interestingly, a study in rural China found that BMI more than $28.0 \mathrm{~kg} / \mathrm{m}^{2}$ was independent risk factor of latent tuberculosis infection (LTBI, adjusted odds ratio (aOR) 1.17, 95\% CI 1.04-1.33) [13]. In summary, BMI have an influence on the infection of MTB strains, and it may also affect the infection of resistant strains. However, no previous publications seem to have examined the impact of different BMI levels on primary drug resistance among TB cases [7-9].

This research intended to explore the association between BMI and primary DR-TB in the aspects as follows: (1) to describe the clinical characteristics of $\mathrm{TB}$ cases with four different BMI status (underweight/normal weight/overweight/obese); (2) to illustrate the drug resistant profiles of $\mathrm{TB}$ cases subgroups stratified by $\mathrm{BMI}$; (3) to analyze the relative risk of DR-TB including DR-TB (total), MDR-TB (total), mono-resistant tuberculosis (MR-TB, total), polydrug resistant tuberculosis (PDR-TB, total), RFP-related resistance, INH-related resistance, streptomycin (SM)-related resistance, MR-TB $(\mathrm{INH}), \mathrm{INH}+\mathrm{RFP}+\mathrm{SM}$ resistance (MDR3), $\mathrm{INH}+\mathrm{SM}$ resistance (PDR2), Any INH + SM resistance among subgroups with different BMI; (4) to investigate the risk factors of DR-TB among TB cases subgroups stratified by BMI. 


\section{Methods}

\section{Statement}

The Ethics Committee of Shandong Provincial Hospital $(\mathrm{SPH})$ and Shandong Provincial Chest Hospital (SPCH) approved for our study. Personal information of TB patients such as names were erased before data analysis and reporting. All methods were performed in accordance with relevant guidelines and regulations. Informed consent was obtained from all participants or, if participants are under 18 , from a parent and/or legal guardian.

\section{Setting}

Our study was conducted in Shandong, a coastal province in eastern China $\left(36^{\circ} 24^{\prime} \mathrm{N}\right.$ latitude and $118^{\circ} 24^{\prime} \mathrm{E}$ longitude), with 100 million inhabitants and an area of $157,100 \mathrm{~km}^{2}$ [14]. A large-scale nationwide survey found that 211,900 population in Shandong suffered from TB in 2010 [15], and its incidence had reduced from 40.8 to 26.25 per 100,000 from 2005 to 2017 [16]. The rates of MR-TB, MDR-TB, PDR-TB among newly diagnosed TB cases in Shandong during 2018 were 13.35\%, 3.73\%, $4.30 \%$, respectively [17]. As estimated, the overall standardized prevalence of combined overweight and obesity among Chinese adults ( $\geq 40$ years) were $30.3 \%$ (95\% CI $30.1-30.4)$, and the prevalence of obesity (BMI $\geq 30 \mathrm{~kg} /$ $\mathrm{m}^{2}$ ) was $3.5 \%$ (95\% CI 3.4-3.6) [18].

\section{Data collection and definitions}

8957 newly diagnosed pulmonary TB patients with BMI status and DST results were collected from 34 monitoring sites of DR-TB in Shandong, SPH and SPCH from 1 Jan 2004 to 31 Dec 2019 retrospectively. Only patients with positive sputum smear specimens from 34 monitoring sites in Shandong were enrolled, and then these positive specimens were sent to the laboratory center in SPCH for strain identification and drug susceptibility tests (DST), meanwhile their basic demography and clinical characteristics were collected. Information of BMI, age, sex (male or female), drinking (yes or no), smoking (yes or no), cavity (yes or no), and co-morbidity were collected through questionnaire. Nutritional status indicators except BMI were not routinely collected. Exclusion criteria: (1) cases with previous TB history; (2) Nontuberculosis mycobacteria (NTM) infection; (3) BMI information missing; (4) extra-pulmonary cases (Fig. 1). We excluded extra-pulmonary TB patients because there were only 60 patients with extra-pulmonary tuberculosis, and those patients were more complicated than pulmonary TB patients.

BMI below $18.5 \mathrm{~kg} / \mathrm{m}^{2}$ were in the underweight range, between 18.5 and $24.9 \mathrm{~kg} / \mathrm{m}^{2}$ were in normal weight range, between 25 and $29.9 \mathrm{~kg} / \mathrm{m}^{2}$ were in the overweight range, $\geq 30 \mathrm{~kg} / \mathrm{m}^{2}$ were in the obese range [19].

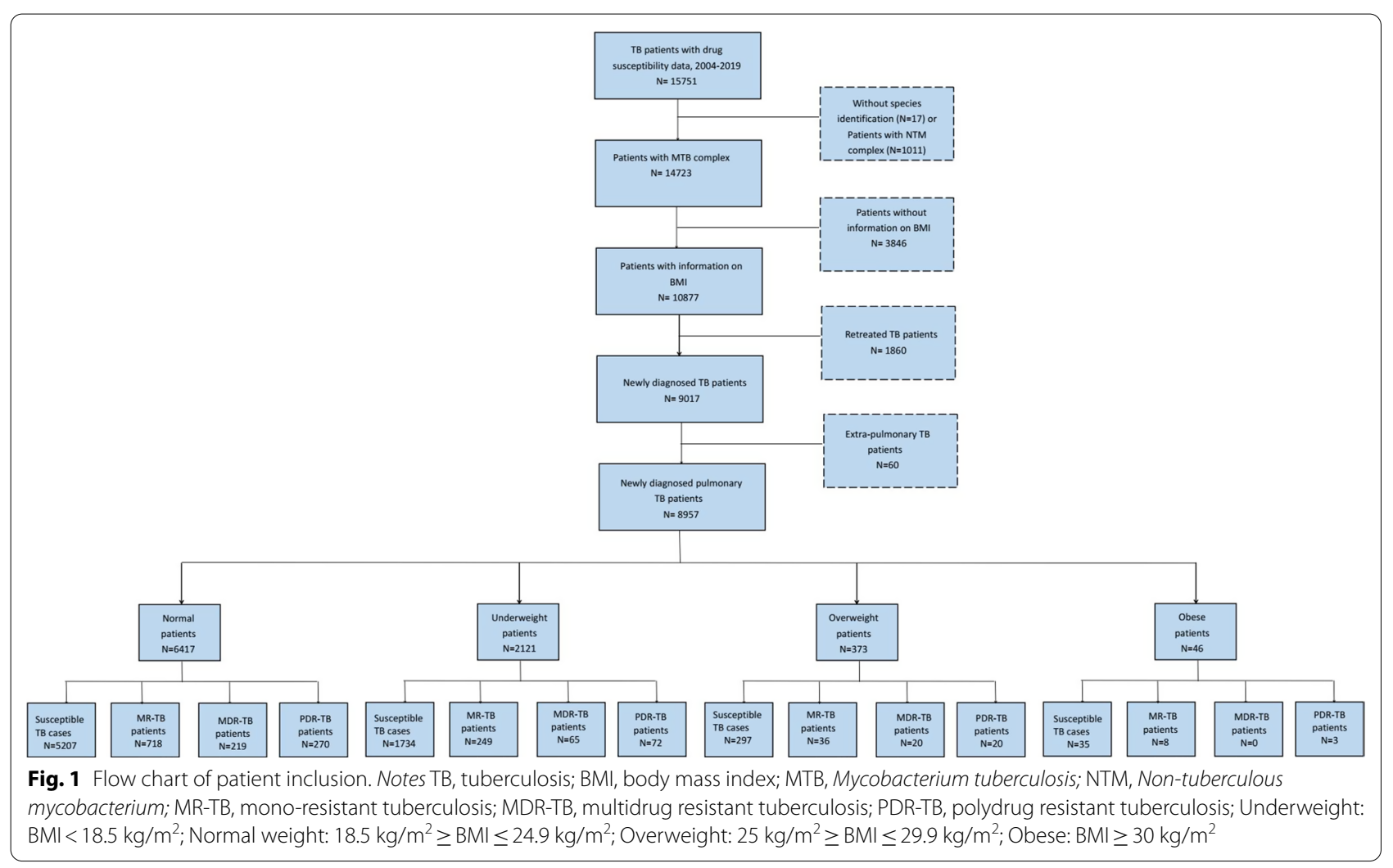


Mono-resistance (MR) was defined as resistance to only one anti-TB drug of first-line [20]. Multidrug resistance (MDR) refers to resistance to at least both INH and RFP [20]. Polydrug resistance (PDR) was defined as resistance to at least two anti-TB drugs of first-line, but except resistance to both INH and RFP [20]. MR-TB (INH) refers to TB patients who are resistant only to isoniazid and sensitive to other drugs. INH-related resistance was defined as resistance to at least isoniazid.

\section{Laboratory methods}

Bacteriological culture, strain identification, and phenotypic DST were all conducted in Katharine Hsu International Research Center of Human Infectious Diseases (KICID) of SPCH. Initially, at least two sputum specimens of each eligible patient were collected. Then, isolates were cultured in Löwenstein-Jensen (L-J) medium according to the standard protocol, and then these growing colonies were used for strain identification and phenotypic DST [17]. DST for four anti-TB drugs of first-line (INH, $0.2 \mu \mathrm{g} / \mathrm{mL}$; RFP, $40 \mu \mathrm{g} / \mathrm{mL}$; SM, $10 \mu \mathrm{g} / \mathrm{mL}$; ethambutol (EMB), $2 \mu \mathrm{g} / \mathrm{mL}$ were routinely conducted through absolute concentration method on L-J media. Standard traditional biochemical testing such as $16 \mathrm{~S}$ rRNA gene sequence analysis were applied for strain identification [17]. The above steps were completed independently by at least two professionally trained investigators, and Superior TB National Reference laboratory in SPCH was responsible for external quality assessment.

\section{Data analysis}

Categorical baseline demographic and clinical features of 8957 TB cases including age (0-14, 15-24, 25-44, 45-64, $65+$ ), sex (men or women), smoker or non-smoker, drinker or non-drinker, cavity (yes/no), co-morbidity (yes/no), and drug resistant profiles in four subgroups with different BMI status were compared by Pearson Chi-square test or Fisher's exact test. The age subgroups were divided according to previous publications [21, 22]. Binary logistic regression models including univariable and multivariable analysis were applied to investigate the correlation between BMI status and various drug resistant sub-types. Due to the mutually exclusive outcomes of MR, MDR, and PDR, we also carried out univariable and multivariable multinomial logistic regression analysis. Moreover, logistic regression models were also used to estimate the risk factors of primary drug resistance among normal weight, overweight, or obese TB group, respectively. According to published studies, categorical covariates including age, sex, smoking status, alcohol use, cavity (yes/no), co-morbidity were adjusted for multivariable analysis [17]. Statistically significant refers to a two-sided $P$ value $<0.05$. All data analyses were conducted in SPSS software (version 20.0).

\section{Results \\ Patients' characteristics}

As shown in Table 1, 8957 newly diagnosed pulmonary TB patients with DST results and BMI status were enrolled in our analysis, of which 6417 (71.64\%) were normal weight, 2121 (23.68\%) were underweight, 373 (4.16\%) were overweight, and $46(0.51 \%)$ were obese. Of the overall patients, $15.62 \%$ aged between 15 and 24 , $25.31 \%$ aged between 25 and 44,33.16\% aged between 45 and $64,25.63 \%$ aged more than $65,83.01 \%$ were males, $10.95 \%$ have co-morbidity such as diabetes (6.84\%), chronic obstructive pulmonary disease (COPD, 1.88\%), hypertension $(6.84 \%)$, HIV/AIDS or other immunocompromised diseases $(7 / 8957,0.08 \%)$.

\section{Underweight TB cases}

Compared with normal BMI cases, TB cases with lower BMI are more likely to be in $15-24$ (17.44\% vs $15.63 \%)$ or $>65$ (34.64\% vs $22.98 \%)$ age group, and they had a lower rate of co-morbidity $(8.44 \%$ vs $10.57 \%), P<0.05$. Underweight TB cases had a higher proportion of asthma $(0.85 \%$ vs $0.37 \%)$ and COPD $(2.55 \%$ vs $1.78 \%)$ but with a lower rate of diabetes $(3.58 \%$ vs $7.03 \%)$ than normal group, $P<0.05$. There were no significant differences in sex, cavity, smoking and drinking between lower and normal BMI cases. Both these two groups have more males, but less drinkers, smokers, and cavity (Table 1).

\section{Overweight TB cases}

The proportions of $25-44$ age group (33.33\% vs $15.63 \%)$, $45-64$ age group $(39.78 \%$ vs $34.21 \%)$, cavity (51\% vs $43.66 \%)$, co-morbidity $(19.57 \%$ vs $10.57 \%)$, COPD (3.22\% vs $1.78 \%)$, diabetes ( $13.94 \%$ vs $7.03 \%)$, hypertension $(3.75 \%$ vs $1.82 \%)$, and cancer $(0.80 \%$ vs $0.16 \%)$ were higher in overweight TB cases than in normal weight cases, $P<0.05$. In addition, overweight $\mathrm{TB}$ cases are less likely to be in $15-24$ age group $(6.18 \%$ vs $15.63 \%)$, $\mathrm{P}<0.05$. There were no significant differences in $0-14$ age group, 65+age group, sex, smoking and drinking between overweight and normal cases (Table 1).

\section{Obese TB cases}

The rates of females ( $32.61 \%$ vs $17.19 \%$ ), co-morbidity (23.91\% vs $10.57 \%)$, diabetes $(19.57 \%$ vs $7.03 \%)$ were higher in obese TB cases than in normal cases, $\mathrm{P}<0.05$. There were no significant differences in age, smoking, drinking and cavity between obese and normal cases (Table 1). 
Table 1 Baseline characteristics of 8957 newly diagnosed pulmonary TB patients in Shandong, China, 2004-2019

\begin{tabular}{|c|c|c|c|c|c|c|c|c|}
\hline \multirow[t]{2}{*}{ Characteristics } & \multirow{2}{*}{$\begin{array}{l}\text { Total } \\
(\mathrm{n}=8957)\end{array}$} & \multicolumn{4}{|c|}{ BMI category $\left(\mathrm{kg} / \mathrm{m}^{2}\right)$} & \multicolumn{3}{|l|}{$P$ value } \\
\hline & & $\begin{array}{l}\text { Normal } \\
\text { weight } \\
\text { TB cases } \\
(n=6417)\end{array}$ & $\begin{array}{l}\text { Underweight } \\
\text { TB cases } \\
(\mathrm{n}=2121)\end{array}$ & $\begin{array}{l}\text { Overweight } \\
\text { TB cases } \\
(n=373)\end{array}$ & $\begin{array}{l}\text { Obese TB } \\
\text { cases }(n=46)\end{array}$ & $\begin{array}{l}\text { Underweight } \\
\text { group vs } \\
\text { normal weight } \\
\text { group }\end{array}$ & $\begin{array}{l}\text { Overweight } \\
\text { group vs } \\
\text { normal weight } \\
\text { group }\end{array}$ & $\begin{array}{l}\text { Obese group } \\
\text { vs normal } \\
\text { weight group }\end{array}$ \\
\hline \multicolumn{9}{|l|}{ Age (years) } \\
\hline $0-14$ & $\begin{array}{l}25 / 8920 \\
(0.28 \%)\end{array}$ & $\begin{array}{l}12 / 6392 \\
(0.19 \%)\end{array}$ & $\begin{array}{l}11 / 2110 \\
(0.52 \%)\end{array}$ & 1/372 (0.27\%) & $1 / 46(2.17 \%)$ & $0.011^{*}$ & 0.521 & 0.089 \\
\hline $15-24$ & $\begin{array}{l}1393 / 8920 \\
(15.62 \%)\end{array}$ & $\begin{array}{l}999 / 6392 \\
(15.63 \%)\end{array}$ & $\begin{array}{l}368 / 2110 \\
(17.44 \%)\end{array}$ & 23/372 (6.18\%) & $3 / 46(6.52 \%)$ & $0.049^{*}$ & $<0.001^{* * *}$ & 0.102 \\
\hline $25-44$ & $\begin{array}{l}2258 / 8920 \\
(25.31 \%)\end{array}$ & $\begin{array}{l}1725 / 6392 \\
(26.99 \%)\end{array}$ & $\begin{array}{l}393 / 2110 \\
(18.63 \%)\end{array}$ & $\begin{array}{l}124 / 372 \\
(33.33 \%)\end{array}$ & 16/46 (34.78\%) & $<0.001^{* * *}$ & $0.008^{* *}$ & 0.236 \\
\hline $45-64$ & $\begin{array}{l}2958 / 8920 \\
(33.16 \%)\end{array}$ & $\begin{array}{l}2187 / 6392 \\
(34.21 \%)\end{array}$ & $\begin{array}{l}607 / 2110 \\
(28.77 \%)\end{array}$ & $\begin{array}{l}148 / 372 \\
(39.78 \%)\end{array}$ & 16/46 (34.78\%) & $<0.001^{* * *}$ & $0.028^{*}$ & 0.936 \\
\hline$>65$ & $\begin{array}{l}2286 / 8920 \\
(25.63 \%)\end{array}$ & $\begin{array}{l}1469 / 6392 \\
(22.98 \%)\end{array}$ & $\begin{array}{l}731 / 2110 \\
(34.64 \%)\end{array}$ & $\begin{array}{l}76 / 372 \\
(20.43 \%)\end{array}$ & 10/46 (21.74\%) & $<0.001^{* * *}$ & 0.254 & 0.842 \\
\hline \multicolumn{9}{|l|}{ Sex } \\
\hline Female & $\begin{array}{l}1522 / 8957 \\
(16.99 \%)\end{array}$ & $\begin{array}{l}1103 / 6417 \\
(17.19 \%)\end{array}$ & $\begin{array}{l}339 / 2121 \\
(15.98 \%)\end{array}$ & $\begin{array}{l}65 / 373 \\
(17.47 \%)\end{array}$ & 15/46 (32.61\%) & 0.199 & 0.906 & $0.006^{* *}$ \\
\hline Male & $\begin{array}{l}7435 / 8957 \\
(83.01 \%)\end{array}$ & $\begin{array}{l}5314 / 6417 \\
(82.81 \%)\end{array}$ & $\begin{array}{l}1782 / 2121 \\
(84.02 \%)\end{array}$ & $\begin{array}{l}308 / 373 \\
(82.8 \%)\end{array}$ & $31 / 46$ (67.39\%) & 0.199 & 0.906 & $0.006^{* *}$ \\
\hline \multicolumn{9}{|l|}{ Cavity } \\
\hline No & $\begin{array}{l}4501 / 8013 \\
(56.17 \%)\end{array}$ & $\begin{array}{l}3205 / 5689 \\
(56.34 \%)\end{array}$ & $\begin{array}{l}1102 / 1931 \\
(57.07 \%)\end{array}$ & $171 / 349(49 \%)$ & $23 / 44(52.27 \%)$ & 0.575 & $0.007^{* *}$ & 0.588 \\
\hline Yes & $\begin{array}{l}3512 / 8013 \\
(43.83 \%)\end{array}$ & $\begin{array}{l}2484 / 5689 \\
(43.66 \%)\end{array}$ & $\begin{array}{l}829 / 1931 \\
(42.93 \%)\end{array}$ & 178/349 (51\%) & $21 / 44(47.73 \%)$ & 0.575 & $0.007^{* *}$ & 0.588 \\
\hline \multicolumn{9}{|l|}{ Smoking } \\
\hline No & $\begin{array}{l}5400 / 7224 \\
(74.75 \%)\end{array}$ & $\begin{array}{l}3847 / 5137 \\
(74.89 \%)\end{array}$ & $\begin{array}{l}1266 / 1725 \\
(73.39 \%)\end{array}$ & $\begin{array}{l}261 / 328 \\
(79.57 \%)\end{array}$ & 26/34 (76.47\%) & 0.217 & 0.057 & 0.832 \\
\hline Yes & $\begin{array}{l}1824 / 7224 \\
(25.25 \%)\end{array}$ & $\begin{array}{l}1290 / 5137 \\
(25.11 \%)\end{array}$ & $\begin{array}{l}459 / 1725 \\
(26.61 \%)\end{array}$ & $\begin{array}{l}67 / 328 \\
(20.43 \%)\end{array}$ & $8 / 34(23.53 \%)$ & 0.217 & 0.057 & 0.832 \\
\hline \multicolumn{9}{|l|}{ Drinking } \\
\hline No & $\begin{array}{l}5663 / 7174 \\
(78.94 \%)\end{array}$ & $\begin{array}{l}4015 / 5013 \\
(80.09 \%)\end{array}$ & $\begin{array}{l}1353 / 1708 \\
(79.22 \%)\end{array}$ & $\begin{array}{l}266 / 329 \\
(80.85 \%)\end{array}$ & 29/34 (85.29\%) & 0.435 & 0.738 & 0.449 \\
\hline Yes & $\begin{array}{l}1511 / 7174 \\
(21.06 \%)\end{array}$ & $\begin{array}{l}1088 / 5013 \\
(21.7 \%)\end{array}$ & $\begin{array}{l}355 / 1708 \\
(20.78 \%)\end{array}$ & $\begin{array}{l}63 / 329 \\
(19.15 \%)\end{array}$ & 5/34 (14.71\%) & 0.435 & 0.738 & 0.449 \\
\hline \multicolumn{9}{|l|}{ Co-morbidity } \\
\hline Total & $\begin{array}{l}941 / 8957 \\
(10.95 \%)\end{array}$ & $\begin{array}{l}678 / 6417 \\
(10.57 \%)\end{array}$ & $\begin{array}{l}179 / 2121 \\
(8.44 \%)\end{array}$ & $\begin{array}{l}73 / 373 \\
(19.57 \%)\end{array}$ & $11 / 46(23.91 \%)$ & $0.005^{* *}$ & $<0.001^{* * *}$ & $0.003^{* *}$ \\
\hline Asthma & $\begin{array}{l}44 / 8957 \\
(0.51 \%)\end{array}$ & $\begin{array}{l}24 / 6417 \\
(0.37 \%)\end{array}$ & $\begin{array}{l}18 / 2121 \\
(0.85 \%)\end{array}$ & 2/373 (0.54\%) & $0 / 46(0.00 \%)$ & $0.007^{* *}$ & 0.652 & 1.000 \\
\hline COPD & $\begin{array}{l}181 / 8957 \\
(2.11 \%)\end{array}$ & $\begin{array}{l}114 / 6417 \\
(1.78 \%)\end{array}$ & $\begin{array}{l}54 / 2121 \\
(2.55 \%)\end{array}$ & $12 / 373(3.22 \%)$ & $1 / 46(2.17 \%)$ & $0.027^{* *}$ & $0.045^{*}$ & 0.563 \\
\hline Bronchiectasis & $\begin{array}{l}35 / 8957 \\
(0.41 \%)\end{array}$ & $\begin{array}{l}24 / 6417 \\
(0.37 \%)\end{array}$ & $\begin{array}{l}11 / 2121 \\
(0.52 \%)\end{array}$ & 0/373 (0.00\%) & $0 / 46(0 \%)$ & 0.366 & 0.640 & 1.000 \\
\hline Silicosis & $\begin{array}{l}27 / 8957 \\
(0.31 \%)\end{array}$ & $\begin{array}{l}24 / 6417 \\
(0.37 \%)\end{array}$ & $3 / 2121(0.14 \%)$ & 0/373 (0.00\%) & $0 / 46(0.00 \%)$ & 0.119 & 0.640 & 1.000 \\
\hline Diabetes & $\begin{array}{l}588 / 8957 \\
(6.84 \%)\end{array}$ & $\begin{array}{l}451 / 6417 \\
(7.03 \%)\end{array}$ & $\begin{array}{l}76 / 2121 \\
(3.58 \%)\end{array}$ & $\begin{array}{l}52 / 373 \\
(13.94 \%)\end{array}$ & 9/46 (19.57\%) & $<0.001^{* * *}$ & $<0.001^{* * *}$ & $<0.001^{* * *}$ \\
\hline Hypertension & $\begin{array}{l}162 / 8957 \\
(1.88 \%)\end{array}$ & $\begin{array}{l}117 / 6417 \\
(1.82 \%)\end{array}$ & $\begin{array}{l}29 / 2121 \\
(1.37 \%)\end{array}$ & 14/373 (3.75\%) & 2/46 (4.35\%) & 0.160 & $0.017^{*}$ & 0.207 \\
\hline Cancer & $\begin{array}{l}19 / 8957 \\
(0.22 \%)\end{array}$ & $\begin{array}{l}10 / 6417 \\
(0.16 \%)\end{array}$ & $6 / 2121(0.28 \%)$ & $3 / 373(0.8 \%)$ & 0/46 (0\%) & 0.241 & $0.031^{*}$ & 1.000 \\
\hline $\begin{array}{l}\text { HIV/AIDS or other } \\
\text { immunocompro- } \\
\text { mised diseases }\end{array}$ & $7 / 8957$ (0.08\%) & $5 / 6417(0.08 \%)$ & 2/2121 (0.09\%) & 0/373 (0\%) & $0 / 46(0 \%)$ & 0.687 & 1.000 & 1.000 \\
\hline
\end{tabular}


Table 1 (continued)

TB, tuberculosis; BMI, body mass index; COPD, chronic obstructive pulmonary disease; HIV/AIDS, human immunodeficiency virus/acquired immune deficiency syndrome

${ }^{*} \mathrm{P}<0.05$; ${ }^{* * \mathrm{P}}<0.01 ;{ }^{* * * \mathrm{P}}<0.001$;

Underweight: $\mathrm{BMI}<18.5 \mathrm{~kg} / \mathrm{m}^{2} ;$ Normal weight: $18.5 \mathrm{~kg} / \mathrm{m}^{2} \geq \mathrm{BMI} \leq 24.9 \mathrm{~kg} / \mathrm{m}^{2} ;$ Overweight: $25 \mathrm{~kg} / \mathrm{m}^{2} \geq \mathrm{BMI} \leq 29.9 \mathrm{~kg} / \mathrm{m}^{2} ;$ obese: $\mathrm{BMI} \geq 30 \mathrm{~kg} / \mathrm{m}^{2}$

\section{Drug resistant profiles}

As shown in Table 2, the proportions of drug resistance among normal weight, underweight, overweight, obese TB groups were $18.86 \% / 18.25 \% / 20.38 \% / 23.91 \%$ (DR-TB),

$$
11.19 \% / 11.74 \% / 9.65 \% / 17.39 \%
$$

(MRTB), $\quad 3.41 \% / 3.06 \% / 5.36 \% / 0.00 \% \quad$ (MDR-TB), $4.21 \% / 3.39 \% / 5.36 \% / 6.52 \%$ (PDR-TB). Underweight group had a lower rate of SM-resistance $(11.6 \%$ vs $13.45 \%)$ but higher rate of MR-TB (INH) (4.34\% vs $3.26 \%)$ than normal weight group, $P<0.05$. Moreover, there were more MDR-TB (5.36\% vs $3.41 \%)$ or MDR3 (INH+RFP + SM)
(3.22\% vs $1.68 \%)$ cases among overweight group than normal group, $P<0.05$. There were no significant differences in other drug resistant sub-types between abnormal weight groups and normal group.

\section{Association between $\mathrm{BMI}$ and primary drug resistance}

Table 3 shows the results of univariable and multivariable analysis of the association between BMI and primary anti-tuberculosis resistance. Compared with normal weight group, underweight were associated with lower risk of SM-related resistance (OR 0.844, 95\%

Table 2 Drug-resistant profiles among newly diagnosed pulmonary TB patients with different BMI

\begin{tabular}{|c|c|c|c|c|c|c|c|}
\hline \multirow[t]{2}{*}{ Drug resistance } & \multirow{2}{*}{$\begin{array}{l}\text { Normal weight } \\
\text { DR-TB cases } \\
(1210 / 6417 \text {, } \\
18.86 \%)\end{array}$} & \multirow{2}{*}{$\begin{array}{l}\text { Underweight } \\
\text { DR-TB cases } \\
(387 / 2121 \text {, } \\
18.25 \%)\end{array}$} & \multirow{2}{*}{$\begin{array}{l}\text { Overweight } \\
\text { DR-TB cases } \\
(76 / 373 \text {, } \\
20.38 \%)\end{array}$} & \multirow{2}{*}{$\begin{array}{l}\text { Obese DR-TB } \\
\text { cases }(11 / 46, \\
23.91 \%)\end{array}$} & \multicolumn{3}{|l|}{$P$ value } \\
\hline & & & & & $\begin{array}{l}\text { Underweight } \\
\text { group vs } \\
\text { Normal weight } \\
\text { group }\end{array}$ & $\begin{array}{l}\text { Overweight } \\
\text { group vs } \\
\text { Normal } \\
\text { weight group }\end{array}$ & $\begin{array}{l}\text { Obese group vs } \\
\text { Normal weight } \\
\text { group }\end{array}$ \\
\hline DR-TB & $1210(18.86 \%)$ & 387 (18.25\%) & $76(20.38 \%)$ & $11(23.91 \%)$ & 0.532 & 0.467 & 0.383 \\
\hline \multicolumn{8}{|l|}{$\begin{array}{l}\text { Any resistance to first- } \\
\text { line drug }\end{array}$} \\
\hline $\mathrm{INH}$ & $663(10.33 \%)$ & $218(10.28 \%)$ & $47(12.6 \%)$ & $5(10.87 \%)$ & 0.944 & 0.164 & 0.905 \\
\hline RFP & 311 (4.85\%) & 94 (4.43\%) & 23 (6.17\%) & 0 (0.00\%) & 0.436 & 0.252 & 0.171 \\
\hline $\mathrm{EMB}$ & $95(1.48 \%)$ & $31(1.46 \%)$ & $6(1.61 \%)$ & $0(0.00 \%)$ & 0.950 & 0.842 & 1.000 \\
\hline SM & $863(13.45 \%)$ & $246(11.6 \%)$ & $60(16.09 \%)$ & $9(19.57 \%)$ & $0.028^{*}$ & 0.149 & 0.226 \\
\hline MR-TB (Total) & $718(11.19 \%)$ & $249(11.74 \%)$ & $36(9.65 \%)$ & $8(17.39 \%)$ & 0.488 & 0.358 & 0.184 \\
\hline INH & 209 (3.26\%) & $92(4.34 \%)$ & $9(2.41 \%)$ & $2(4.35 \%)$ & $0.019^{*}$ & 0.369 & 0.662 \\
\hline RFP & $58(0.9 \%)$ & $18(0.85 \%)$ & $1(0.27 \%)$ & $0(0.00 \%)$ & 0.815 & 0.379 & 1.000 \\
\hline $\mathrm{EMB}$ & $12(0.19 \%)$ & $5(0.24 \%)$ & $1(0.27 \%)$ & $0(0.00 \%)$ & 0.662 & 0.521 & 1.000 \\
\hline SM & $436(6.79 \%)$ & $130(6.13 \%)$ & $25(6.7 \%)$ & $6(13.04 \%)$ & 0.286 & 0.945 & 0.094 \\
\hline Others & $3(0.05 \%)$ & $4(0.19 \%)$ & $0(0.00 \%)$ & $0(0.00 \%)$ & 0.069 & 1.000 & 1.000 \\
\hline MDR-TB (Total) & $219(3.41 \%)$ & $65(3.06 \%)$ & $20(5.36 \%)$ & $0(0.00 \%)$ & 0.438 & $0.047^{*}$ & 0.407 \\
\hline MDR1: INH+RFP & $48(0.75 \%)$ & $15(0.71 \%)$ & $4(1.07 \%)$ & $0(0.00 \%)$ & 0.849 & 0.531 & 1.000 \\
\hline $\begin{array}{l}\text { MDR2: } \\
I N H+R F P+E M B+S M\end{array}$ & 48 (0.75\%) & 16 (0.75\%) & $4(1.07 \%)$ & $0(0.00 \%)$ & 0.977 & 0.531 & 1.000 \\
\hline MDR3: INH+RFP + SM & $108(1.68 \%)$ & $30(1.41 \%)$ & $12(3.22 \%)$ & $0(0.00 \%)$ & 0.395 & $0.029^{*}$ & 1.000 \\
\hline Others & $15(0.23 \%)$ & $4(0.19 \%)$ & $0(0.00 \%)$ & $0(0.00 \%)$ & 1.000 & 1.000 & 1.000 \\
\hline$P D R-T B$ & $270(4.21 \%)$ & $72(3.39 \%)$ & $20(5.36 \%)$ & $3(6.52 \%)$ & 0.098 & 0.284 & 0.444 \\
\hline PDR1: INH+EMB & $6(0.09 \%)$ & $2(0.09 \%)$ & $1(0.27 \%)$ & $0(0 \%)$ & 1.000 & 0.327 & 1.000 \\
\hline PDR2: INH+SM & $219(3.41 \%)$ & $55(2.59 \%)$ & $17(4.56 \%)$ & $3(6.52 \%)$ & 0.063 & 0.241 & 0.209 \\
\hline PDR3: RFP + SM & $27(0.42 \%)$ & $8(0.38 \%)$ & $2(0.54 \%)$ & $0(0.00 \%)$ & 0.785 & 0.672 & 1.000 \\
\hline PDR4: INH+EMB + SM & $10(0.16 \%)$ & $3(0.14 \%)$ & $0(0.00 \%)$ & $0(0.00 \%)$ & 1.000 & 1.000 & 1.000 \\
\hline Others & $8(0.12 \%)$ & $4(0.19 \%)$ & $0(0.00 \%)$ & $0(0.00 \%)$ & 0.507 & 1.000 & 1.000 \\
\hline
\end{tabular}

TB, tuberculosis; DR-TB, drug-resistant tuberculosis; MR-TB, mono-resistant tuberculosis; MDR-TB, multidrug resistant tuberculosis; PDR-TB, polydrug resistant tuberculosis; EMB, ethambutol; INH, isoniazid; RFP, rifampin; SM, streptomycin; BMI, body mass index;* $\mathrm{P}<0.05$ 
Table 3 Association between BMI and primary anti-tuberculosis resistance

\begin{tabular}{|c|c|c|c|c|}
\hline \multirow[t]{2}{*}{ The type of drug resistance } & \multicolumn{2}{|l|}{ Univariable analysis } & \multicolumn{2}{|c|}{ Multivariable analysis } \\
\hline & OR $(95 \% \mathrm{Cl})$ & P value & aOR $(95 \% \mathrm{Cl})$ & P value \\
\hline \multicolumn{5}{|c|}{ Underweight group vs Normal weight group } \\
\hline $\mathrm{DR}-\mathrm{TB}$ & $0.96(0.846-1.09)$ & 0.532 & $0.977(0.86-1.111)$ & 0.726 \\
\hline MR-TB & $1.041(0.893-1.215)$ & 0.606 & $1.056(0.904-1.233)$ & 0.493 \\
\hline MDR-TB & $0.891(0.672-1.182)$ & 0.424 & $0.928(0.698-1.235)$ & 0.610 \\
\hline PDR-TB & $0.801(0.614-1.044)$ & 0.101 & $0.804(0.615-1.052)$ & 0.112 \\
\hline INH-related resistance & $0.994(0.846-1.169)$ & 0.944 & $0.948(0.746-1.203)$ & 0.659 \\
\hline RFP-related resistance & $0.91(0.719-1.153)$ & 0.436 & $0.948(0.746-1.203)$ & 0.659 \\
\hline SM-related resistance & $0.844(0.726-0.982)$ & $0.028^{*}$ & $0.869(0.746-1.012)$ & 0.071 \\
\hline MR-TB (INH) & $1.347(1.049-1.730)$ & $0.020^{*}$ & $1.31(1.017-1.686)$ & $0.037^{*}$ \\
\hline $\mathrm{INH}+\mathrm{SM}$ resistance & $0.754(0.558-1.019)$ & 0.066 & $0.751(0.554-1.018)$ & 0.065 \\
\hline $\mathrm{INH}+\mathrm{RFP}+\mathrm{SM}$ resistance & $0.834(0.555-1.255)$ & 0.384 & $0.881(0.585-1.328)$ & 0.546 \\
\hline Any $\mathrm{INH}+\mathrm{SM}$ resistance & $0.818(0.656-1.019)$ & 0.073 & $0.831(0.665-1.039)$ & 0.105 \\
\hline Susceptible & Reference & Reference & Reference & Reference \\
\hline \multicolumn{5}{|c|}{ Overweight group vs Normal weight group } \\
\hline DR-TB & $1.101(0.849-1.428)$ & 0.467 & $1.082(0.832-1.407)$ & 0.559 \\
\hline MR-TB & $0.879(0.617-1.253)$ & 0.476 & $0.854(0.596-1.225)$ & 0.391 \\
\hline MDR-TB & $1.603(1.002-2.566)$ & $0.049^{*}$ & $1.639(1.02-2.633)$ & $0.041^{*}$ \\
\hline PDR-TB & $1.299(0.812-2.076)$ & 0.275 & $1.284(0.8-2.06)$ & 0.300 \\
\hline INH-related resistance & $1.251(0.912-1.717)$ & 0.165 & $1.248(0.907-1.716)$ & 0.174 \\
\hline RFP-related resistance & $1.29(0.833-1.997)$ & 0.253 & $1.271(0.817-1.977)$ & 0.287 \\
\hline SM-related resistance & $1.234(0.927-1.641)$ & 0.149 & $1.224(0.916-1.636)$ & 0.171 \\
\hline MR-TB (INH) & $0.734(0.374-1.443)$ & 0.371 & $0.736(0.373-1.449)$ & 0.375 \\
\hline $\mathrm{INH}+\mathrm{SM}$ resistance & $1.361(0.82-2.26)$ & 0.234 & $1.36(0.815-2.268)$ & 0.239 \\
\hline $\mathrm{INH}+\mathrm{RFP}+\mathrm{SM}$ resistance & $1.948(1.061-3.577)$ & $0.032^{*}$ & $2.113(1.141-3.912)$ & $0.017^{*}$ \\
\hline Any $\mathrm{INH}+\mathrm{SM}$ resistance & $1.472(1.013-2.14)$ & $0.043^{*}$ & $1.483(1.017-2.164)$ & $0.041^{*}$ \\
\hline Susceptible & Reference & Reference & Reference & Reference \\
\hline \multicolumn{5}{|c|}{ Obese group vs Normal weight group } \\
\hline DR-TB & $0.739(0.374-1.46)$ & 0.384 & $0.741(0.374-1.469)$ & 0.391 \\
\hline MR-TB & $1.658(0.766-3.587)$ & 0.199 & $1.62(0.747-3.516)$ & 0.222 \\
\hline MDR-TB & - & - & - & - \\
\hline PDR-TB & $1.653(0.505-5.409)$ & 0.406 & $1.712(0.52-5.637)$ & 0.377 \\
\hline INH-related resistance & $0.945(0.372-2.399)$ & 0.905 & $0.943(0.37-2.403)$ & 0.901 \\
\hline RFP-related resistance & - & - & - & - \\
\hline SM-related resistance & $0.639(0.307-1.328)$ & 0.230 & $0.644(0.308-1.347)$ & 0.243 \\
\hline MR-TB (INH) & $1.350(0.325-5.607)$ & 0.679 & $1.304(0.313-5.435)$ & 0.715 \\
\hline $\mathrm{INH}+\mathrm{SM}$ resistance & $2.038(0.622-6.678)$ & 0.240 & $2.089(0.633-6.892)$ & 0.226 \\
\hline $\mathrm{INH}+\mathrm{RFP}+\mathrm{SM}$ resistance & - & - & - & - \\
\hline Any INH + SM resistance & $1.136(0.348-3.709)$ & 0.833 & $1.148(0.349-3.775)$ & 0.820 \\
\hline Susceptible & Reference & Reference & Reference & Reference \\
\hline
\end{tabular}

TB, tuberculosis; DR-TB, drug-resistant tuberculosis; MR-TB, mono-resistant tuberculosis; MDR-TB, multidrug resistant tuberculosis; PDR-TB, polydrug resistant tuberculosis; $\mathrm{EMB}$, ethambutol; INH, isoniazid; $\mathrm{RFP}$, rifampin; $\mathrm{SM}$, streptomycin; $\mathrm{BMI}$, body mass index; aOR, adjusted odds ratio; OR, odds ratio; ${ }^{*} \mathrm{P}<0.05$ Underweight: $\mathrm{BMI}<18.5 \mathrm{~kg} / \mathrm{m}^{2}$; Normal weight: $18.5 \mathrm{~kg} / \mathrm{m}^{2} \geq \mathrm{BMI} \leq 24.9 \mathrm{~kg} / \mathrm{m}^{2} ;$ Overweight: $25 \mathrm{~kg} / \mathrm{m}^{2} \geq \mathrm{BMI} \leq 29.9 \mathrm{~kg} / \mathrm{m}^{2} ;$ Obese: $\mathrm{BMI} \geq 30 \mathrm{~kg} / \mathrm{m}^{2}$

CI $0.726-0.982, P=0.028)$, but contributed to a higher risk of MR-TB (INH) (OR 1.347, 95\% CI 1.049-1.730, $P=0.020 ;$ aOR $1.31,95 \%$ CI $1.017-1.686, P=0.037$ ). In addition, overweight was positively associated with MDR-TB (OR 1.603, 95\% CI 1.002-2.566, $P=0.049$; aOR
1.639, 95\% CI 1.02-2.633, $P=0.041), \mathrm{INH}+\mathrm{RFP}+\mathrm{SM}$ resistance (OR 1.948, 95\% CI 1.061-3.577, $P=0.032$; aOR 2.113, 95\% CI 1.141-3.912, $P=0.017)$, Any INH + SM resistance (OR 1.472, 95\% CI 1.013-2.14, $P=0.043$; aOR 1.483, 95\% CI 1.017-2.164, $P=0.041)$. However, there 
were no statistical differences on drug resistance between obese group and normal weight group. In univariable and multivariable multinomial logistic regression models, we also found that overweight may be a risk factor for MDRTB (OR 1.601, 95\% CI 0.998-2.568, $P=0.051$; aOR 1.536, 95\% CI 0.952-2.476, $P=0.078$ ) (see Table 7 in Appendix).

\section{Risk factors for drug resistance}

As shown in Tables 4 and 5, male was a risk factor of DR-TB in underweight (OR 1.385, 95\% CI 1.001-1.917, $P=0.049$; aOR $1.458,95 \%$ CI $1.038-2.048, P=0.030$ ) and overweight cases (OR 2.392, 95\% CI 1.044-5.479, $\mathrm{P}=0.039$; aOR 2.611, 95\% CI 1.107-6.158, $P=0.028$ ). In addition, co-morbidity except COPD, diabetes, hypertension (aOR 4.34, 95\% CI 1.053-17.928, $P=0.042$ ) were positively associated with DR-TB in underweight cases. Among normal weight group, male (aOR 1.26, 95\% CI 1.059-1.513, $\mathrm{P}=0.010$ ) or cavitary (aOR 1.15, 95\% CI 1.006-1.319, $\mathrm{P}=0.030$ ) pulmonary tuberculosis were more likely to have anti-TB resistance (Table 6). We did not find a significant association between factors including age, smoking, drinking, COPD, diabetes, hypertension and drug resistance among underweight, overweight or normal weight group.

\section{Discussion}

Identifying novel risk factors or predictors for DR-TB will facilitate the implementation of $\mathrm{TB}$ termination strategies, but so far little studies have explored the relationship between different TB drug-resistance and BMI status. Our study was designed to compare clinical features and drug-resistant profiles of primary pulmonary TB cases with different BMI, thus to provide reference in clinics. We now report DST results and BMI status of 8957 newly diagnosed pulmonary TB patients, and found that abnormal BMI was related to the elevated risk of some drug-resistant sub-types. Meanwhile, BMI were positively associated with the incidence of accompanying diseases among TB cases. Male was a risk factor of DR-TB in underweight, overweight, or normal TB patients.

Table 4 Univariable and multivariable analysis of risk factors for DR-TB in underweight TB cases

\begin{tabular}{|c|c|c|c|c|c|c|}
\hline \multirow[t]{2}{*}{ Characteristics } & \multirow[t]{2}{*}{ Non-DR $n=1734(\%)$} & \multirow[t]{2}{*}{ DR-TB $n=387(\%)$} & \multicolumn{2}{|l|}{ Univariable analysis } & \multicolumn{2}{|c|}{ Multivariable analysis } \\
\hline & & & OR $(95 \% \mathrm{Cl})$ & P value & OR $(95 \% \mathrm{Cl})$ & P value \\
\hline \multicolumn{7}{|c|}{ Age (years) $(n=1725 / n=385)$} \\
\hline $0-14$ & $9(0.52 \%)$ & $2(0.52 \%)$ & $0.963(0.203-4.557)$ & 0.962 & $1.022(0.213-4.898)$ & 0.978 \\
\hline $15-24$ & $299(17.33 \%)$ & $69(17.92 \%)$ & Reference & Reference & Reference & Reference \\
\hline $25-44$ & $322(18.67 \%)$ & $71(18.44 \%)$ & $0.955(0.662-1.379)$ & 0.808 & $0.984(0.68-1.424)$ & 0.932 \\
\hline $45-64$ & $479(27.77 \%)$ & $128(33.25 \%)$ & $1.158(0.835-1.605)$ & 0.379 & $1.138(0.813-1.594)$ & 0.45 \\
\hline$>65$ & $616(35.71 \%)$ & $115(29.87 \%)$ & $0.809(0.582-1.124)$ & 0.206 & $0.789(0.561-1.109)$ & 0.173 \\
\hline \multicolumn{7}{|l|}{$\operatorname{Sex}(n=1734 / n=387)$} \\
\hline Female & $290(16.72 \%)$ & $49(12.66 \%)$ & Reference & Reference & Reference & Reference \\
\hline Male & $1444(83.28 \%)$ & $338(87.34 \%)$ & $1.385(1.001-1.917)$ & $0.049^{*}$ & $1.458(1.038-2.048)$ & $0.030^{*}$ \\
\hline \multicolumn{7}{|c|}{ Cavity $(n=1566 / n=365)$} \\
\hline No & $896(57.22 \%)$ & $206(56.44 \%)$ & Reference & Reference & Reference & Reference \\
\hline Yes & $670(42.78 \%)$ & $159(43.56 \%)$ & $1.032(0.82-1.299)$ & 0.787 & $1.019(0.807-1.286)$ & 0.875 \\
\hline \multicolumn{7}{|c|}{ Smoking $(n=1423 / n=302)$} \\
\hline No & $1046(73.51 \%)$ & $220(72.85 \%)$ & Reference & Reference & Reference & Reference \\
\hline Yes & 377 (26.49\%) & $82(27.15 \%)$ & $0.77(0.581-1.019)$ & 0.067 & $1.157(0.802-1.669)$ & 0.434 \\
\hline \multicolumn{7}{|c|}{ Drinking $(n=1407 / n=301)$} \\
\hline No & $1108(78.75 \%)$ & $245(81.4 \%)$ & Reference & Reference & Reference & Reference \\
\hline Yes & $299(21.25 \%)$ & $56(18.6 \%)$ & $0.841(0.639-1.107)$ & 0.216 & $0.686(0.455-1.033)$ & 0.071 \\
\hline \multicolumn{7}{|c|}{ Co-morbidity $(n=1734 / n=387)$} \\
\hline No & 1584 (91.35\%) & $358(92.51 \%)$ & Reference & Reference & Reference & Reference \\
\hline COPD & $47(2.71 \%)$ & $7(1.81 \%)$ & $0.661(0.297-1.474)$ & 0.312 & $0.962(0.32-2.892)$ & 0.944 \\
\hline Diabetes & $63(3.63 \%)$ & $13(3.36 \%)$ & $0.922(0.502-1.693)$ & 0.793 & $0.959(0.517-1.779)$ & 0.895 \\
\hline Hypertension & $23(1.33 \%)$ & $6(1.55 \%)$ & $1.172(0.474-2.897)$ & 0.732 & $1.315(0.522-3.316)$ & 0.561 \\
\hline Other co-morbidity & $97(5.59 \%)$ & $15(3.88 \%)$ & $0.68(0.391-1.186)$ & 0.174 & $0.746(0.347-1.604)$ & 0.454 \\
\hline
\end{tabular}

TB, tuberculosis; DR-TB, drug-resistant tuberculosis; aOR, adjusted odds ratio; $\mathrm{OR}$, odds ratio; $\mathrm{COPD}$, chronic obstructive pulmonary disease ${ }^{*} \mathrm{P}<0.05$; Underweight: $\mathrm{BMl}<18.5 \mathrm{~kg} / \mathrm{m}^{2}$ 
Table 5 Univariable and multivariable analysis of risk factors for DR-TB in overweight TB cases

\begin{tabular}{|c|c|c|c|c|c|c|}
\hline \multirow[t]{2}{*}{ Characteristics } & \multirow[t]{2}{*}{ Non-DR $n=297(\%)$} & \multirow[t]{2}{*}{ DR-TB $n=76(\%)$} & \multicolumn{2}{|c|}{ Univariable analysis } & \multicolumn{2}{|c|}{ Multivariable analysis } \\
\hline & & & OR $(95 \% \mathrm{Cl})$ & $P$ value & OR $(95 \% \mathrm{Cl})$ & $P$ value \\
\hline \multicolumn{7}{|c|}{ Age (years) $(n=297 / n=75)$} \\
\hline $0-14$ & $1(0.34 \%)$ & $0(0 \%)$ & NA & NA & NA & NA \\
\hline $15-24$ & $18(6.06 \%)$ & $5(6.67 \%)$ & Reference & Reference & Reference & Reference \\
\hline $25-44$ & $99(33.33 \%)$ & $25(33.33 \%)$ & $0.909(0.308-2.687)$ & 0.863 & $0.687(0.224-2.11)$ & 0.512 \\
\hline $45-64$ & $113(38.05 \%)$ & $35(46.67 \%)$ & $1.115(0.386-3.221)$ & 0.841 & $0.84(0.278-2.534)$ & 0.756 \\
\hline$>65$ & $66(22.22 \%)$ & $10(13.33 \%)$ & $0.545(0.165-1.799)$ & 0.319 & $0.434(0.121-1.557)$ & 0.2 \\
\hline \multicolumn{7}{|l|}{$\operatorname{Sex}(n=297 / n=76)$} \\
\hline Female & $58(19.53 \%)$ & $7(9.21 \%)$ & Reference & Reference & Reference & Reference \\
\hline Male & $239(80.47 \%)$ & $69(90.79 \%)$ & $2.392(1.044-5.479)$ & $0.039^{*}$ & $2.611(1.107-6.158)$ & $0.028^{*}$ \\
\hline \multicolumn{7}{|c|}{ Cavity $(n=281 / n=68)$} \\
\hline No & $142(50.53 \%)$ & $29(42.65 \%)$ & Reference & Reference & Reference & Reference \\
\hline Yes & 139 (49.47\%) & $39(57.35 \%)$ & $1.374(0.805-2.345)$ & 0.244 & $1.298(0.736-2.287)$ & 0.367 \\
\hline \multicolumn{7}{|c|}{ Smoking $(n=259 / n=69)$} \\
\hline No & 204 (78.76\%) & $57(82.61 \%)$ & Reference & Reference & Reference & Reference \\
\hline Yes & 55 (21.24\%) & $12(17.39 \%)$ & $1.517(0.643-3.577)$ & 0.341 & $0.743(0.321-1.724)$ & 0.49 \\
\hline \multicolumn{7}{|c|}{ Drinking $(n=260 / n=69)$} \\
\hline No & $207(79.62 \%)$ & $59(85.51 \%)$ & Reference & Reference & Reference & Reference \\
\hline Yes & $53(20.38 \%)$ & $10(14.49 \%)$ & $0.662(0.317-1.381)$ & 0.271 & $0.629(0.261-1.516)$ & 0.302 \\
\hline \multicolumn{7}{|c|}{ Co-morbidity $(n=297 / n=76)$} \\
\hline No & $239(80.47 \%)$ & $59(77.63 \%)$ & Reference & Reference & Reference & Reference \\
\hline COPD & $10(3.37 \%)$ & $2(2.63 \%)$ & $0.776(0.166-3.617)$ & 0.746 & $0.265(0.031-2.293)$ & 0.228 \\
\hline Diabetes & $41(13.8 \%)$ & $11(14.47 \%)$ & $1.057(0.515-2.169)$ & 0.881 & $1.235(0.563-2.708)$ & 0.599 \\
\hline Hypertension & $12(4.04 \%)$ & $2(2.63 \%)$ & $0.642(0.141-2.931)$ & 0.567 & $0.705(0.138-3.615)$ & 0.676 \\
\hline Other co-morbidity & $16(5.39 \%)$ & $6(7.89 \%)$ & $1.505(0.568-3.987)$ & 0.411 & $4.34(1.053-17.928)$ & $0.042^{*}$ \\
\hline
\end{tabular}

TB, tuberculosis; DR-TB, drug-resistant tuberculosis; aOR, adjusted odds ratio; OR, odds ratio; COPD, chronic obstructive pulmonary disease

*P $<0.05$; Overweight: $25 \mathrm{~kg} / \mathrm{m}^{2} \geq B M I \leq 29.9 \mathrm{~kg} / \mathrm{m}^{2}$

Obesity has become a global public health problem, and it's reported that approximately $46 \%$ of adults and $15 \%$ of children China are overweight or obese [23]. Plenty of evidence have revealed that BMI is negatively associated with active tuberculosis [6, 24]. For instance, a study among 46028 adult participants in Taiwan found that obesity (BMI $\left.\geq 27 \mathrm{~kg} / \mathrm{m}^{2}\right)$ (aOR $0.43 ; 95 \%$ CI $\left.0.28-0.67\right)$ and overweight $\left(\mathrm{BMI}=24-26.9 \mathrm{~kg} / \mathrm{m}^{2}\right)(\mathrm{aOR} 0.67 ; 95 \%$ CI 0.49-0.91) contributed to a lower risk of TB infection [24]. A study in rural China found that BMI $\geq 28.0 \mathrm{~kg} /$ $\mathrm{m}^{2}$ was observed to be independently associated with LTBI (aOR: 1.17, 95\% CI 1.04-1.33) [13]. Interestingly, our study indicated that overweight was a risk factor for MDR-TB, INH + RFP + SM resistance, Any INH + SM resistance. The explanation may be that MTB had a lower virulence due to high-fat exposure and immunoregulation of leptin, and the reproduction speed of MTB with lipid bodies were slower than those without such lipid bodies [25, 26]. Furthermore, MTB can survive without reproducing in fatty tissues, and may go some way towards explaining why higher BMI contributed to LTBI.
As we know, primary drug-resistant TB was caused by the transmission of resistant MTB strains, and this resistance may come at a "fitness cost" through a decreased transmission rate, virulence and reproduction speed [27]. Therefore, we supposed that obesity may have a greater impact on reducing the transmission of susceptible MTB strain than some resistant strains, because the transmission of the resistant strains were already at a lower level due to the "fitness cost", which may explain why there was a higher risk of falling ill with MDR-TB, INH + RFP + SM resistant TB, or Any INH + SM resistant TB among overweight population. Some overweight and obese patients can also be malnourished, since BMI is only one of prognostic factors for assessing malnutrition. Fat accumulation in overweight and obese individuals may induce additional nutritional derangements, both indirectly through acute and chronic diseases with negative impact on nutritional status and directly through metabolic and body composition changes [28]. Furthermore, skeletal muscle mass and function (sarcopenia) may also contribute to malnutrition. Malnutrition in obesity may be an 
Table 6 Univariable and multivariable analysis of risk factors for DR-TB in normal weight cases

\begin{tabular}{|c|c|c|c|c|c|c|}
\hline \multirow[t]{2}{*}{ Characteristics } & \multirow[t]{2}{*}{ Non-DR $n=5207(\%)$} & \multirow[t]{2}{*}{ DR-TB $n=1210(\%)$} & \multicolumn{2}{|l|}{ Univariable analysis } & \multicolumn{2}{|c|}{ Multivariable analysis } \\
\hline & & & OR $(95 \% \mathrm{Cl})$ & $P$ value & OR $(95 \% \mathrm{Cl})$ & $P$ value \\
\hline \multicolumn{7}{|c|}{ Age (years) $(n=5186 / n=1206)$} \\
\hline $0-14$ & $10(0.19 \%)$ & $2(0 \%)$ & $0.868(0.189-3.997)$ & 0.856 & $0.96(0.209-4.459)$ & 0.963 \\
\hline $15-24$ & $812(15.66 \%)$ & $187(0.26 \%)$ & Reference & Reference & Reference & Reference \\
\hline $25-44$ & $1356(26.15 \%)$ & $369(0.52 \%)$ & $1.182(0.971-1.438)$ & 0.096 & $1.17(0.961-1.426)$ & 0.118 \\
\hline $45-64$ & $1780(34.32 \%)$ & $407(0.57 \%)$ & $0.993(0.819-1.203)$ & 0.942 & $0.95(0.781-1.159)$ & 0.622 \\
\hline$>65$ & $1228(23.68 \%)$ & $241(0.34 \%)$ & $0.852(0.69-1.052)$ & 0.137 & $0.829(0.667-1.03)$ & 0.091 \\
\hline \multicolumn{7}{|c|}{$\operatorname{Sex}(n=5207 / n=1210)$} \\
\hline Female & $918(17.63 \%)$ & 185 (15.29\%) & Reference & Reference & Reference & Reference \\
\hline Male & $4289(82.37 \%)$ & $1025(84.71 \%)$ & $1.186(0.998-1.408)$ & 0.052 & $1.26(1.059-1.513)$ & $0.010^{*}$ \\
\hline \multicolumn{7}{|c|}{ Cavity $(n=4624 / n=1065)$} \\
\hline No & $2640(57.09 \%)$ & $565(53.05 \%)$ & Reference & Reference & Reference & Reference \\
\hline Yes & $1984(42.91 \%)$ & $500(46.95 \%)$ & $0.86(0.702-1.054)$ & 0.147 & $1.15(1.006-1.319)$ & $0.040^{*}$ \\
\hline \multicolumn{7}{|c|}{ Smoking $(n=4188 / n=949)$} \\
\hline No & $3125(74.62 \%)$ & $722(76.08 \%)$ & Reference & Reference & Reference & Reference \\
\hline Yes & $1063(25.38 \%)$ & 227 (23.92\%) & $0.924(0.784-1.09)$ & 0.348 & $0.984(0.79-1.226)$ & 0.887 \\
\hline \multicolumn{7}{|c|}{ Drinking $(n=4160 / n=943)$} \\
\hline No & 3257 (78.29\%) & $758(80.38 \%)$ & Reference & Reference & Reference & Reference \\
\hline Yes & $903(21.71 \%)$ & 185 (19.62\%) & $0.88(0.738-1.051)$ & 0.158 & $0.858(0.68-1.084)$ & 0.2 \\
\hline \multicolumn{7}{|c|}{ Co-morbidity $(n=5207 / n=1210)$} \\
\hline No & 4665 (89.59\%) & $1074(88.76 \%)$ & Reference & Reference & Reference & Reference \\
\hline COPD & $94(1.81 \%)$ & $20(1.65 \%)$ & $0.91(0.562-1.487)$ & 0.718 & $0.938(0.5-1.76)$ & 0.843 \\
\hline Diabetes & $354(6.8 \%)$ & $97(8.02 \%)$ & $1.195(0.946-1.51)$ & 0.136 & $1.23(0.969-1.568)$ & 0.089 \\
\hline Hypertension & $98(1.88 \%)$ & $19(1.57 \%)$ & $0.83(0.507-1.365)$ & 0.466 & $0.91(0.548-1.514)$ & 0.72 \\
\hline Other co-morbidity & $216(4.15 \%)$ & $51(4.21 \%)$ & $1.01(0.744-1.389)$ & 0.917 & $1.11(0.746-1.667)$ & 0.596 \\
\hline
\end{tabular}

DR-TB, drug-resistant tuberculosis; aOR, adjusted odds ratio; $\mathrm{OR}$, odds ratio; $\mathrm{COPD}$, chronic obstructive pulmonary disease

${ }^{*} \mathrm{P}<0.05$; Normal weight: $18.5 \mathrm{~kg} / \mathrm{m}^{2} \geq \mathrm{BMl} \leq 24.9 \mathrm{~kg} / \mathrm{m}^{2}$

important factor for some increased resistance among newly-diagnosed tuberculosis patient. The indicators for nutritional status include anthropometric index and laboratory parameters $[29,30]$. The former mainly includes height, weight, body mass index and alternative indices, trunk measurements (waist and hip circumferences and sagittal abdominal diameter) and limb measurements (mid-upper arm and calf circumferences) and skinfold thickness [29], and the latter mainly includes albumin, prealbumin, urinary creatinine or 3-methylhistidine [30]. However, this retrospective study were not available for other nutritional indexes except BMI. Therefore, it may be a good idea to detect more indicators for nutritional status as above among TB cases to find those with both burden of obesity and malnutrition in future, and then explore their drug-resistant profiles.
Overweight TB cases had a higher possibility of suffering from co-morbidity including COPD, hypertension, diabetes, and cancer. Actually, previous studies have figured out that BMI was a reliable predictor of prevalent diabetes, hypertension, and COPD [31-33]. A study in in South Asian cities among 31,118 participants found that every standard deviation higher of BMI was associated with 1.42 and 1.28 times higher probability of hypertension and 1.65 and 1.60 times higher probability of diabetes among 40-69 years men and women respectively [31]. A dose-response relationship between COPD and BMI has been observed among both males and females, for example the prevalence of COPD increased from 2.5 to $7.6 \%$ in men and from 3.5 to $13.4 \%$ in women when their BMI changed from normal to obese (BMI $\geq 40)$ [33]. Recent years, former studies have found that COPD (aOR 
1.86, 95\% CI 1.01-2.93, $\mathrm{P}=0.041)$ and diabetes (aOR $1.59,95 \% \mathrm{CI} 1.04-2.44, \mathrm{P}=0.03)$ could increase the risk of MDR-TB and PDR-TB, respectively [34-36]. Thus, it can be seen that screening for drug resistance among overweight TB cases, especially those combined with COPD or diabetes, may help monitor the development and guide the clinical diagnosis and treatment of DR-TB.

People have found a significant association between underweight and higher risk of active TB, adverse TB treatment outcomes, tuberculosis-related mortality [7, 37-39]. According to a study among the US population in 1971-1975, estimated TB incidence among adults who were underweight, normal, overweight, and obese was 260.2 (95\% CI 98.6, 421.8), 24.7 (95\% CI 13.0-36.3), 8.9 (95\% CI 2.2-15.6), and 5.1 (95\% CI 0.0-10.5) per 100,000 person-years, respectively [38]. Interestingly, we found underweight was associated with lower risk of SM-related resistance and co-morbidity, but contributed to a higher risk of MR-TB (INH). Studies have also shown that underweight is associated with abnormal cellmediated immunity, phagocytic function, complement system, immunoglobulin A secretion, and cytokine production such as reduced secretion of Th1 cytokines (IL-2, TNF- $\alpha$ and interferon- $\gamma$ ), which may lead to more severe TB infection [40]. Moreover, malnourished animals have higher bacterial burdens and impaired immune system (e.g. reduction of reactive $\mathrm{N}$ intermediates) [37, 41], Underweight population may influence the infection INH-resistant or SM-resistant MTB strains through immune system, but the molecular mechanism remains to be further explored. Our findings would provide guidance for clinicians when treating underweight TB cases, for instance, they should maintain keen vigilance at INH resistance rather than SM resistance.

It has been observed that males were more likely to be affected with DR-TB among normal, underweight, or overweight population. However, there were inconsistent results in the previous literature about the influence of gender differences on vulnerability to MDR-TB [42-44]. A study conducted in Lianyungang city, China found females were more likely to be infected with MDRTB (aOR 1.763, 95\% CI 1.060-2.934) [42], while Faustini stated that MDR-TB patients tended to be male (OR 1.38; 95\% CI 1.16-1.65) in western Europe [43]. We assume that the heterogeneity on the susceptibility of DR-TB among males and females may be related to the their differences in airway structure and lifestyles such as smoking, drinking, stay up late.

This study had some strengths. Firstly, it's the first study to investigate the effect of BMI on drug resistance among newly diagnosed pulmonary TB cases. Secondly, the relative risk of numerous drug-resistant sub-types including DR-TB (total), MDR-TB (total), MR-TB (total), PDR-TB (total), RFP-related resistance, INH-related resistance, SM-related resistance, IMR-TB (INH), INH + RFP + SM resistance (MDR3), INH + SM resistance (PDR2), Any $\mathrm{INH}+\mathrm{SM}$ resistance among underweight, overweight, obese were analyzed, and could provide clinical reference for future pathologic and molecular studies on different resistant MTB strains. Thirdly, the large amount (DST results from a province of 100 million people) and long time span (from 2004 to 2019) of our data guaranteed the reliance of our findings.

This study also had some limitations. Firstly, drug resistance of second-line anti-TB drugs were not routinely examined in China unless at the initiative requirements of patients. Thus, the association between BMI and second-line anti-TB resistance remains to be discovered in future. Secondly, this was a retrospective study, and may have information bias. Thirdly, some obese patients can also be malnourished, since BMI is only one of prognostic factors for assessing malnutrition [28, 29, 45]. However, we were not available for other nutritional indexes except BMI due to its retrospective study model. This might be an important confounding factor in the obese group.

\section{Conclusion}

Our study has important implications on the global triple epidemics of underweight, obesity, and DR-TB. The positive effect of overweight on MDR-TB, INH + RFP + SM resistance, Any INH + SM resistance, and co-morbidity implies that routine screening for drug sensitivity and more attention on co-morbidity among overweight TB cases may be necessary. Although patients with normal weight had a lower proportion of drug-resistance than overweight or obese patients in Shandong, screening for drug resistance cannot be ignored either because patients with normal weight accounted for $74.64 \%$. In addition, we found underweight TB cases have a higher risk of INH resistance, which provides a reference for clinical rational use of drugs. Our study suggests that an in-depth study of the interaction between host metabolic activity and infection of DR-TB may contribute more to novel treatment options or preventive measures, and accelerate the implementation of the STOP TB strategy.

\section{Appendix}

See Table 7. 


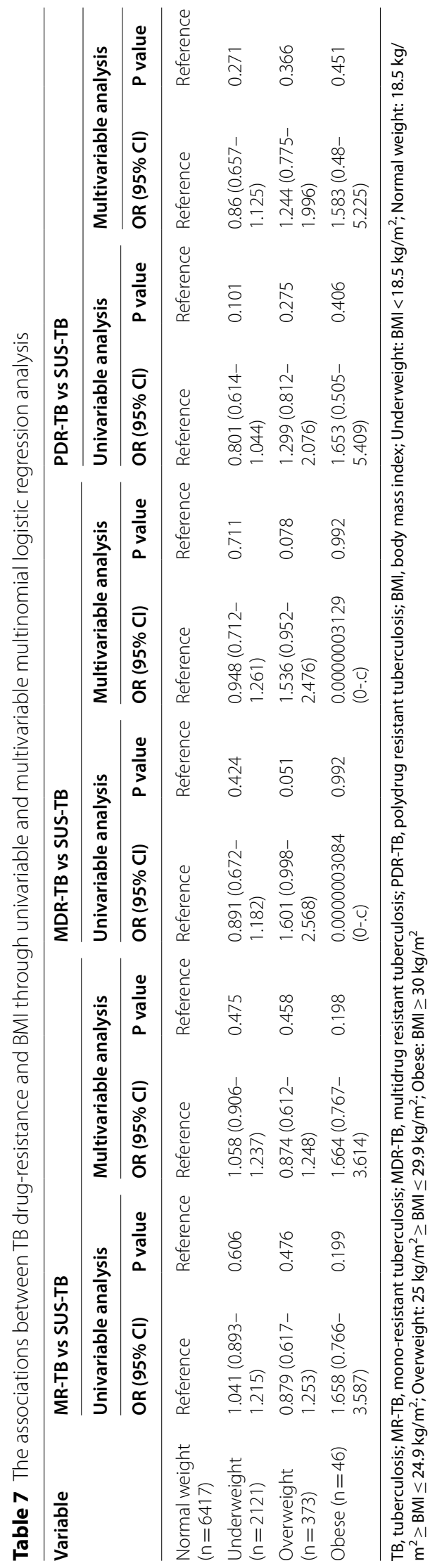




\begin{abstract}
Abbreviations
TB: Tuberculosis; DR-TB: Drug-resistant tuberculosis; WHO: World Health Organization; COPD: Chronic obstructive pulmonary disease; RR-TB: Rifampinresistant tuberculosis; MDR-TB: Multidrug resistant tuberculosis; MR-TB: Mono-resistant tuberculosis; PDR-TB: Polydrug resistant tuberculosis; LTBI: Latent tuberculosis infection; aOR: Adjusted odds ratio; OR: Odds ratio; Cl: Confidence interval; INH: Isoniazide; RFP: Rifampin; EMB: Ethambutol; SM: Streptomycin; MTB: Mycobacterium tuberculosis; DST: Drug susceptibility test; NTM: Non-tuberculous mycobacterium; HIV/AIDS: Human immunodeficiency virus/acquired immune deficiency syndrome.
\end{abstract}

\section{Acknowledgements}

We thank Shandong Provincial Hospital, Shandong Provincial Chest Hospital, 13 municipal-level and 21 county-level local health departments for drug susceptibility data, sociodemographic and clinical data.

\section{Authors' contributions}

HCL, WMS, JG and YFL conceived and designed the study. HCL, CBY, QQA, SQL, CXY and LG directed its implementation including the data analysis and writing of the paper. WMS, JG and YFL analyzed the data; YL, QYZ, JYL, TTX, SJL and NNT contributed materials/analytic tools; WMS, YFL and HCL wrote and revised the manuscript. All authors revised it critically for important intellectual content, gave final approval of the version to be published and agreed to be accountable for all aspects of the work in ensuring that questions related to the accuracy or integrity of any part of the work were appropriately investigated and resolved. All authors read and approved the final manuscript.

\section{Funding}

This work was supported by Natural Science Foundation of Shandong Province (No. ZR2020KH013), Department of Science and Technology of Shandong Province (CN) (Nos. 2007GG30002033; 2017GSF218052) and Jinan Science and Technology Bureau (CN) (No.201704100). The study sponsors had not participated in the study design, in the collection, analysis and interpretation of data; in the writing of the manuscript; and in the decision to submit the manuscript for publication.

\section{Availability of data and materials}

Data can be available through contact with the corresponding author.

\section{Declarations}

\section{Ethics approval and consent to participate}

The protocols applied in this study were approved by the Ethics Committee of Shandong Provincial Hospital, affiliated with Shandong University (SPH) and the Ethic Committee of Shandong Provincial Chest Hospital (SPCH), China. Personal identifiers of TB patients were removed before data analysis and reporting. Informed consent was obtained from all participants or, if participants are under 18, from a parent and/or legal guardian. All methods were performed in accordance with relevant guidelines and regulations.

\section{Consent for publication}

Not applicable.

\section{Competing interests}

The authors declare that they have no competing interests.

\section{Author details}

'Department of Respiratory and Critical Care Medicine, Shandong Provincial Hospital Affiliated to Shandong University, Shandong Provincial Hospital Affiliated to Shandong First Medical University, Jinan 250021, Shandong, People's Republic of China. ${ }^{2}$ Cheeloo College of Medicine, Shandong University, Jinan 250012, Shandong, People's Republic of China. ${ }^{3}$ School of Medicine and Life Sciences, Shandong First Medical University and Shandong Academy of Medical Sciences, Taian 271016, Shandong, People's Republic of China. ${ }^{4}$ Department of Emergency, The Fifth People's Hospital of Jinan, Jinan 250031, Shandong, People's Republic of China. ${ }^{5}$ Department of Intensive Care Unit, Shandong Provincial Third Hospital, Jinan 100191, Shandong, People's Republic of China. ${ }^{6}$ Department of Respiratory and Critical Care Medicine, Beijing Hospital, Beijing 100730, People's Republic of China. ${ }^{7}$ Graduate School of Peking Union Medical College, Chinese Academy of Medical Sciences and Peking Union Medical College, Beijing 100730, People's Republic of China. ${ }^{8}$ Katharine Hsu International Research Center of Human Infectious Diseases, Shandong Provincial Chest Hospital, Jinan 250013, Shandong, People's Republic of China. ${ }^{9}$ Department of Respiratory Medicine, Heze Mudan People's Hospital, Heze 274000, Shandong, People's Republic of China. ${ }^{10} \mathrm{College}$ of Traditional Chinese Medicine, Shandong University of Traditional Chinese Medicine, Jinan 250355, Shandong, People's Republic of China.

Received: 30 March 2021 Accepted: 1 December 2021

Published online: 06 December 2021

\section{References}

1. WHO Global tuberculosis report. https://www.who.int/tb/publications/ global_report/en/

2. Nourzad S, Jenkins HE, Milstein M, Mitnick CD. Estimating the global burden of multidrug-resistant tuberculosis among prevalent cases of tuberculosis. Int J Tuberc Lung Dis. 2017;21(1):6-11.

3. Rajbhandary SS, Marks SM, Bock NN. Costs of patients hospitalized for multidrug-resistant tuberculosis. Int J Tuberc Lung Dis. 2004;8(8):1012-6.

4. Pandey P, Pant ND, Rijal KR, Shrestha B, Kattel S, Banjara MR, Maharjan B, Kc R. Diagnostic accuracy of GeneXpert MTB/RIF assay in comparison to conventional drug susceptibility testing method for the diagnosis of multidrug-resistant tuberculosis. PLoS ONE. 2017;12(1):e0169798.

5. Pradipta IS, Forsman LD, Bruchfeld J, Hak E, Alffenaar JW. Risk factors of multidrug-resistant tuberculosis: a global systematic review and metaanalysis. J Infect. 2018;77(6):469-78.

6. Lin HH, Wu CY, Wang CH, Fu H, Lönnroth K, Chang YC, Huang YT. Association of obesity, diabetes, and risk of tuberculosis: two population-based cohorts. Clin Infect Dis. 2018;66(5):699-705.

7. Yen YF, Chuang PH, Yen MY, Lin SY, Chuang P, Yuan MJ, Ho BL, Chou $P$, Deng CY. Association of body mass index with tuberculosis mortality: a population-based follow-up study. Medicine (Baltimore). 2016:95(1):e2300.

8. Park HO, Kim SH, Moon SH, Byun JH, Kim JW, Lee CE, Kim JD, Jang IS, Yang $\mathrm{JH}$. Association between body mass index and sputum culture conversion among South Korean patients with multidrug resistant tuberculosis in a tuberculosis referral hospital. Infect Chemother. 2016;48(4):317-23.

9. Soh AZ, Chee CBE, Wang YT, Yuan JM, Koh WP. Diabetes and body mass index in relation to risk of active tuberculosis: a prospective populationbased cohort. Int J Tuberc Lung Dis. 2019;23(12):1277-82.

10. Lönnroth K, Jaramillo E, Williams BG, Dye C, Raviglione M. Drivers of tuberculosis epidemics: the role of risk factors and social determinants. Soc Sci Med. 2009;68(12):2240-6.

11. Scrimshaw NS, SanGiovanni JP. Synergism of nutrition, infection, and immunity: an overview. Am J Clin Nutr. 1997;66(2):464s-77s.

12. Falagas ME, Kompoti M. Obesity and infection. Lancet Infect Dis. 2006;6(7):438-46.

13. Zhang H, Li X, Xin H, Li H, Li M, Lu W, Bai L, Wang X, Liu J, Jin Q, et al. Association of body mass index with the tuberculosis infection: a populationbased study among 17796 adults in rural China. Sci Rep. 2017;7:41933.

14. Shandong. https://en.wikipedia.org/wiki/Shandong.

15. Wang L, Zhang H, Ruan Y, Chin DP, Xia Y, Cheng S, Chen M, Zhao Y, Jiang S, Du X, et al. Tuberculosis prevalence in China, 1990-2010; a longitudinal analysis of national survey data. Lancet. 2014;383(9934):2057-64.

16. Tao NN, Li YF, Wang SS, Liu YX, Liu JY, Song WM, Liu Y, Geng H, Li HC. Epidemiological characteristics of pulmonary tuberculosis in Shandong, China, 2005-2017: a retrospective study. Medicine (Baltimore). 2019;98(21):e15778.

17. Song WM, Li YF, Ma XB, Liu JY, Tao NN, Liu Y, Zhang QY, Xu TT, Li SJ, Yu CB, et al. Primary drug resistance of Mycobacterium tuberculosis in Shandong, China, 2004-2018. Respir Res. 2019;20(1):223.

18. Tong X, Wang X, Wang D, Chen D, Qi D, Zhang H, Wang Z, Lu Z, Li W. Prevalence and ethnic pattern of overweight and obesity among middleaged and elderly adults in China. Eur J Prev Cardiol. 2019;26(16):1785-9.

19. Global Health Observatory $(\mathrm{GHO})$ data, Mean Body Mass Index (BMI). https://www.who.int/gho/ncd/risk_factors/bmi text/en/.

20. Falzon D, Schünemann HJ, Harausz E, González-Angulo L, Lienhardt C, Jaramillo E, Weyer K. World Health Organization treatment guidelines for drug-resistant tuberculosis, 2016 update. Eur Respir J. 2017:49(3):1602308 
21. Salindri AD, Wang JY, Lin HH, Magee MJ. Post-tuberculosis incidence of diabetes, myocardial infarction, and stroke: retrospective cohort analysis of patients formerly treated for tuberculosis in Taiwan, 2002-2013. Int J Infect Dis. 2019;84:127-30.

22. Zhang $X$, Andersen AB, Lillebaek T, Kamper-Jørgensen Z, Thomsen $V$, Ladefoged K, Marrs CF, Zhang L, Yang Z. Effect of sex, age, and race on the clinical presentation of tuberculosis: a 15-year population-based study. Am J Trop Med Hyg. 2011;85(2):285-90.

23. Wang $Y$, Xue H, Sun M, Zhu X, Zhao L, Yang Y. Prevention and control of obesity in China. Lancet Glob Health. 2019;7(9):e1166-7.

24. Yen YF, Hu HY, Lee YL, Ku PW, Lin IF, Chu D, Lai YJ. Obesity/overweight reduces the risk of active tuberculosis: a nationwide population-based cohort study in Taiwan. Int J Obes (Lond). 2017;41(6):971-5.

25. Garton NJ, Waddell SJ, Sherratt AL, Lee SM, Smith RJ, Senner C, Hinds J, Rajakumar K, Adegbola RA, Besra GS, et al. Cytological and transcript analyses reveal fat and lazy persister-like bacilli in tuberculous sputum. PLoS Med. 2008;5(4):e75.

26. Neyrolles $\mathrm{O}$, Hernández-Pando R, Pietri-Rouxel F, Fornès $P$, Tailleux $L$, Barrios Payán JA, Pivert E, Bordat Y, Aguilar D, Prévost MC, et al. Is adipose tissue a place for Mycobacterium tuberculosis persistence? PLOS ONE. 2006;1(1):e43.

27. Gagneux S. Fitness cost of drug resistance in Mycobacterium tuberculosis. Clin Microbiol Infect. 2009;15(Suppl 1):66-8.

28. Barazzoni R, Gortan Cappellari G. Double burden of malnutrition in persons with obesity. Rev Endocr Metab Disord. 2020;21(3):307-13.

29. Madden AM, Smith S. Body composition and morphological assessment of nutritional status in adults: a review of anthropometric variables. J Hum Nutr Diet. 2016;29(1):7-25.

30. Keller U. Nutritional laboratory markers in malnutrition. J Clin Med. 2019;8(6):775

31. Patel SA, Ali MK, Alam D, Yan LL, Levitt NS, Bernabe-Ortiz A, Checkley W, Wu Y, Irazola V, Gutierrez L, et al. Obesity and its relation with diabetes and hypertension: a cross-sectional study across 4 geographical regions. Glob Heart. 2016;11(1):71-79.e74.

32. Lambert AA, Putcha N, Drummond MB, Boriek AM, Hanania NA, Kim V, Kinney GL, McDonald MN, Brigham EP, Wise RA, et al. Obesity is associated with increased morbidity in moderate to severe COPD. Chest. 2017;151(1):68-77.

33. Fuller-Thomson E, Howden KEN, Fuller-Thomson LR, Agbeyaka S. A strong graded relationship between level of obesity and COPD: findings from a national population-based study of lifelong nonsmokers. J Obes. 2018;2018:6149263.

34. Song WM, Li YF, Liu JY, Tao NN, Liu Y, Zhang QY, Xu TT, Li SJ, An QQ, Liu $\mathrm{SQ}$, et al. Drug resistance of previously treated tuberculosis patients with diabetes mellitus in Shandong, China. Respir Med. 2020;163:105897.

35. Zhao JN, Zhang XX, He XC, Yang GR, Zhang XQ, Xin WG, Li HC. Multidrugresistant tuberculosis in patients with chronic obstructive pulmonary disease in China. PLoS ONE. 2015;10(8):e0135205.

36. Song WM, Shao Y, Liu JY, Tao NN, Liu Y, Zhang QY, Xu TT, Li SJ, Yu CB, Gao L, et al. Primary drug resistance among tuberculosis patients with diabetes mellitus: a retrospective study among 7223 cases in China. Infect Drug Resist. 2019;12:2397-407.

37. Yen YF, Tung FI, Ho BL, Lai YJ. Underweight increases the risk of early death in tuberculosis patients. Br J Nutr. 2017;118(12):1052-60.

38. Cegielski JP, Arab L, Cornoni-Huntley J. Nutritional risk factors for tuberculosis among adults in the United States, 1971-1992. Am J Epidemiol. 2012;176(5):409-22.

39. Kornfeld H, Sahukar SB, Procter-Gray E, Kumar NP, West K, Kane K, Natarajan M, Li W, Babu S, Viswanathan V. Impact of diabetes and low body mass index on tuberculosis treatment outcomes. Clin Infect Dis. 2020;71(9):e392-8.

40. Dobner JKS. Body mass index and the risk of infection-from underweight to obesity. Clin Microbiol Infect. 2018;24(1):24-8.

41. Cegielski JP, McMurray DN. The relationship between malnutrition and tuberculosis: evidence from studies in humans and experimental animals. Int J Tuberc Lung Dis. 2004;8(3):286-98.

42. Liu Q, Zhu L, Shao Y, Song H, Li G, Zhou Y, Shi J, Zhong C, Chen C, Lu W. Rates and risk factors for drug resistance tuberculosis in Northeastern China. BMC Public Health. 2013;13:1171.

43. Faustini A, Hall AJ, Perucci CA. Risk factors for multidrug resistant tuberculosis in Europe: a systematic review. Thorax. 2006;61(2):158-63.
44. Mor Z, Goldblatt D, Kaidar-Shwartz H, Cedar N, Rorman E, Chemtob D. Drug-resistant tuberculosis in Israel: risk factors and treatment outcomes. Int J Tuberc Lung Dis. 2014;18(10):1195-201.

45. Leibovitz E, Giryes S, Makhline R, Zikri Ditch M, Berlovitz Y, Boaz M. Malnutrition risk in newly hospitalized overweight and obese individuals: $\mathrm{Mr}$ NOI. Eur J Clin Nutr. 2013;67(6):620-4.

\section{Publisher's Note}

Springer Nature remains neutral with regard to jurisdictional claims in published maps and institutional affiliations.
Ready to submit your research? Choose BMC and benefit from:

- fast, convenient online submission

- thorough peer review by experienced researchers in your field

- rapid publication on acceptance

- support for research data, including large and complex data types

- gold Open Access which fosters wider collaboration and increased citations

- maximum visibility for your research: over $100 \mathrm{M}$ website views per year

At BMC, research is always in progress.

Learn more biomedcentral.com/submissions 